\title{
The Attribution of Unity of Consciousness over Time
}

\author{
Seyyed Bahram Borgheai \\ University of Rhode Island, Kingston, USA \\ Email: borgheai@uri.edu
}

How to cite this paper: Borgheai, S. B. (2019). The Attribution of Unity of Consciousness over Time. Open Journal of Philosophy, 9, 1-14.

https://doi.org/10.4236/ojpp.2019.91001

Received: November 15, 2018

Accepted: December 25, 2018

Published: December 28, 2018

Copyright $\odot 2019$ by author and Scientific Research Publishing Inc. This work is licensed under the Creative Commons Attribution International License (CC BY 4.0).

http://creativecommons.org/licenses/by/4.0/

\section{c) (i) Open Access}

\begin{abstract}
There is hardly a consensus over attribution of unity to consciousness in terms of experience duration. Mainly, there are two major accounts of the unity over the course of time: diachronic and synchronic. However, the main problem with each of these stances is transitivity problem; that is, one cannot stand with one of these accounts without admitting the other. The way that Tye describes his diachronic account of unity is transitive to the synchronic type. Similarly, Bayne's confinement to mere synchronic unity faces transitivity problem from synchronic to diachronic type. Dainton's co-consciousness, also, fails to offer a coherent account to include both synchronic and diachronic unities. To resolve the problems around unity attribution over time, a holistic view, here called "network unity" model is proposed. Using the network account, a coherent scheme of unity relation can be achieved in such a way that both synchronic and diachronic unities can be subsumed by a single conception.
\end{abstract}

\section{Keywords}

Consciousness, Synchronic and Diachronic Unities, Co-Consciousness, Extensionalism and Retentionalism

\section{Introduction}

There seems to be a working consensus among philosophers about the phenomenal unity of consciousness. We, the mindful creatures, experience our modalities (experience tokens) phenomenally ${ }^{1}$ in a single unified manner. In the conjunctive account, the unity relation is defined as follows: for a subject $S$ suppose 1) $S$ has an $F$ experience (at $t), 2$ ) $S$ has a $G$ experience (at $t)$, then the conjunc-

${ }^{1}$ Like Bayne (2010), the type of unity discussed here is the phenomenal and not the representational nor subject unity. 
tive unity thesis will be that 3) $\mathrm{S}$ has an " $\mathrm{F}$ and G" experience (at t), i.e. an experience constructed out of the conjunction of $\mathrm{F}$ and $\mathrm{G}$. This way of presenting the unity relation-that is, to describe it as some relation/property at a specific time t-tempts us to consider unity as an instantaneous relation, a type of relation in each instant of our experience. Technically, this type of unity is called synchronic unity. While some philosophers such as Bayne (2010) are proponents of this type of unity for consciousness, there are those such as Tye (2003) and Dainton (2006) who attribute the unity to more extended periods of time. Dainton generalizes the relation from synchronic to diachronic while Tye, and more radically, posits an awake stream of consciousness (from one unconscious state to another) as a single unified experience.

There is, thus, a controversy over attribution of unity based on duration of experience. Some, like Bayne, concentrate only on the synchronic type, while Dainton advocates both types of synchronic and diachronic but in different senses. For his part, Tye is inclined to take the whole conscious awake stream as a unified experience.

This attribution of unity over time is the focus of this paper. We investigate how the accounts of unity work in different durations of experience. As discussed later, the main problem in attribution of unity over time is that the accounts of synchronic and diachronic unity affect each other. In other words, the way one deals with one type of unity is transitive to the other type. Let us call this the "transitivity problem". This problem appears when it is supposed that picking only one type of unity entails rejecting or neglecting the other, as proponents of synchronic only or diachronic only do. Even embracing both types (as Dainton does) is not sufficient. To remove the transitivity problem, there should be a similar or consistent account of both types of unity-otherwise, when the accounts of two unity types are dissimilar, then, by transitivity, we will face two dissimilar accounts for each synchronic and diachronic unities. We know this as the "dissimilarity problem".

In this paper, we first discuss different stances taken by Tye, Bayne and Dainton and then examine how their accounts work in attribution of unity over time, especially when facing the transitivity and dissimilarity problems. As none of these accounts seem successful in resolving the problems, a new proposal will be suggested which embraces both synchronic and diachronic types in such a way that there will be less concern about transitivity and dissimilarity problems. In addition, embracing both types by a uniform framework, theoretically, helps to clarify the concept of consciousness unity in a more explanatory manner (Masrour, forthcoming). This may ultimately allow us to discuss the relation between the one-ness of the subject and the unity of consciousness.

Before considering how different accounts of unity deal with synchronous and diachronic types, it should be clarified what the referent of the term "synchronic" is. Normally, "synchronic" means to "be at the same instant of time", and for experiences one should take "instant" as a "subjective instant". When we want to 
discuss any type of attribution over time, the unit of time should be clear. Subsequently, in determining the attribution of unity of consciousness, as a phenomenal entity, one should explain how the subjective instant relates to the physical instant, as an objective unit of time. Is there any difference between the subjective and objective instants? If yes, how this difference affects the attribution of unity of consciousness over time?

\section{Subjective Instantaneity}

For each (physical) moment, if only one instantaneous experience is available, then some sorts of experience, such as movement, persistence, change or passage cannot be explained ${ }^{2}$, because for these experiences we need to have more than one instantaneous representation in our subjective experience to elicit its transient characteristic.

To resolve this explanatory problem, there are two approaches to temporal perception, in particular the "nowness" or present perception: Extensionalism and Retentionalism. These differ in their position on the duration of the subjective instant and in their explanation of how the information of past is available at present. According to Extensionalism, the subjective instant is not just a point-like representation of some precise objective moment, but rather the mind gathers data from different physical moments in a (very) brief period of time based on which subjective moment is experienced. This short-extended period is called the "specious present" which represents "now". Accordingly, the instantaneous subjective moment has some duration and is not point-like (Dainton, 2006: p. 138). The idea was originated with William James' writings (1890), developed later by C. D. Broad (1923), and is recently advocated by Dainton, among others.

Retentionalists, on the other hand, explain the experience of passage and motion in a different manner. The idea, primarily proposed by Husserl, is that our subjective instant is point-like but has some "retention" of the past (and future) based on which experience of the present, motion and change is constructed.

There is some empirical evidence related to this debate. Experiments show that if we stimulate a subject with two successive incidences with a brief lag, the subject feels both incidences occurring as a single stimulus ${ }^{3}$. This means that there might be no one-to-one correspondence between objective moment and subjective instant. The "phi phenomenon" is further evidence of this occurrence. In this experiment two differently colored dots-for example, one green and one red-will be exposed to a subject with $150 \mathrm{~ms}$ lag, where each stimulus persists for $50 \mathrm{~ms}$. Subjects observe these dots as one dot moving from one to another and, more surprisingly, having a color change during the path (Kolers \& Gru-

${ }^{2}$ Here, I have taken the realist account of these experiences, i.e. to claim that we experience distinguishable phenomenal characteristics in transitions and movements; it is not mere knowledge or inference of them.

${ }^{3}$ For visual stimuli, this period is less than $20 \mathrm{~ms}$ (as in film), 2 - $3 \mathrm{~ms}$ for auditory, and $10 \mathrm{~ms}$ for tactile (Ruhnau, 1995). 
nau, 1976). In addition, the color change demonstrates that in the representation of the middle of the path, there is some knowledge and process of the future.

Here, I do not intend to discuss which of these two approaches is the more plausible. My goal, rather, is to postulate a common general principle, acceptable to both views, which can be applied in our discourse of unity over time. According to the above evidence, I am inclined to stipulate the Non-Correspondence Principle as follows:

Non-Correspondence Principle: there is no one-to-one correspondence between subjective instants and objective moments.

This means that it is not the case that every subjective instant at $t$ corresponds to only one condition in the physical world at (the objective moment of) t. Past and future are present in our "now" experience. The mind aggregates data from the past and future and then, after some processing, the representation of an instant emerges; that is, a group or set of physical states (and not one state only) are attributed to a subjective moment. Thus, subjective instantaneity is not identical to objective physical synchrony.

Based on this background, we can now discuss how unity accounts work over time.

\section{Existing Approaches toward Unity over Time}

There are three possible positions to take for the unity of consciousness over time: only diachronic unity; only synchronous unity; and embracing both. For the first, we have selected Tye's position to describe, for the second Bayne's and Dainton's views, and for the third we will discuss the holism view, chiefly advocated by Bayne and Dainton.

\subsection{Tye's Position}

Using a top-down approach, Tye (2003) takes a single continuous awake conscious stream (i.e. between two successive unconscious states) as a unit (minimum constitutive element) of unified experience, for instance between two successive sleeps. He starts from the top because for him it is problematic to start from instantaneous experience tokens and build a unified experience from them. Tye does not accept that the total unified experience is made of experience tokens. He evades the problem of unity construction from individuals-i.e. experience tokens-by claiming that this construction is meaningless. In other words, for Tye, the synchronous unity as building blocks of the persisted experienced unity is not acceptable. In contrast, his preference is the diachronic type, i.e. the prolonged awake/conscious period.

Consequently, for him, it is challenging to clarify the nature of unity relation. For, he rejects the presence of relata in unity relation (i.e. experience tokens) without which, it is hard to conceive of any relation. Tye believes a unified experience possesses an "overall specific PANIC (Poised Abstract Non-Conceptual Intentional Character)" and thus a specific phenomenal character (2003: p. 99), 
while, at the same time, admits that each moment possesses a specific PANIC. Here, we can see the transitivity problem. The way Tye treats the diachronic unity-i.e. possessing a specific PANIC-is also applicable to the synchronous unity. Even if he accepts both types and ignores the individuation problem, he should clarify how an "overall PANIC" for a diachronic unity is related to the PANICs of individual synchronous unities. So, there is some sort of dissimilarity problem, here, in relating the two types over their PANICs. Therefore, Tye faces the problem of transitivity from the whole awake unity (diachronic) to the synchronic unity.

\subsection{Bayne and Dainton's Synchronic Unity}

In contrast to Tye, many others such as Bayne, Dainton and Hurley (1998) see experience tokens as the mere subject of unified relation and so admit the synchronous unity. Bayne (2010) concentrates only on the synchronic unity, stating his unity thesis as: "Necessarily, for any conscious subject of experience (S) and any time $(t)$, the simultaneous conscious states that $S$ has at $t$ will be subsumed by a single conscious state-the subject's total conscious state" (2010: p. 16). For him, relata are simultaneous conscious states or experience tokens (in contrast to Tye) and the relation is subsumption. On discussing his unity thesis, Bayne takes only the synchronic unity into consideration: "The idea, roughly, is that any 'instantaneous snapshot' of a subject's experience will reveal it to be fully unified" (2010: p. 17). He supposes experiences as being "states that can be enjoyed 'all at once"' (2010: p. 23) or "a slice of the stream of consciousness" (2010: p. 17).

It should be noted that Bayne posits an objective and not a subjective conception of time as the criterion for cutting this slice: "The temporal framework in question is clock-like, not that of the content of experience" (2010: p. 18). And it seems that he posits an objective "all at once" physical instant to be a one-to-one correspondent to (or even identical with) each "slice of conscious stream". This contrasts with our initially introduced NCP (Nonlinear Complementarity Principle). Empirically, there is no such correspondence; there is no objective "all at once" experience. Besides, what Bayne supposes to be one slice of a conscious stream should be construed as a subjective moment, since it is a cut of "conscious" experience. Therefore, it should be the experienced moment and not the "clock-like" moment; the unity thesis, as he claims, is about every instant of our experience. Thus, his conception of "synchronous" is insufficiently precise. Moreover, if one grants the "specious present" stance, then each subjective moment has some duration, and since there will be no "all at once" moment, it would be hard to recognize the synchronous unity in Bayne's framework. There is no clear criteria to distinguish synchronic from diachronic.

Moreover, Bayne's criteria for the individuation of experience are problematic and transitive to the diachronic type of unity. Bayne (2010) introduces a "tripartite conception" for individuation of experiences: subject, time, and phenomenal 
properties. One can enumerate many durable and non-instantaneous experiences which share a common phenomenal character and are confined to a specific time. Melodies, motion, a movie experience, an evening with a friend, a football match, or even Tye's day-long experience-all are examples of an infinite set of durable experiences which meet tripartite criteria: they are all attributed to one mindful subject, share a common phenomenal character, and also related to a specific time. Thus, the tripartite conception also holds for non-instantaneous unities in longer durations. In other words, his criteria are transitive from his only accepted synchronic type to a durable diachronic one.

Bayne's views on the nature of unity relation encounter the same transitivity problem. For Bayne, unity relation is subsumption (Bayne \& Chalmers, 2003), which, according to Hempel and Oppenheim (1948), is covered under the same law (rule or regularity). It is conceivable to claim that each of the above non-instantaneous examples of experience is subsumed by a single or common rule which relates them to a single event. Hence, again, the subsumption criterion is transitive to diachronic unity.

Dainton (2006), on the other hand, goes deeper to clarify the unity relation. He interprets unity as a co-conscious relation, i.e. being together in a single conscious experience. For him, co-presence is an essential condition for the synchronic unity. For a unity relation, the relata, i.e. experience tokens, should already be present together. He then introduces a new concept for unity which is "maximal connection": "an experience is maximally connected if all its distinguishable parts are mutually and equally co-conscious" (2006: p. 105). With this maximal connection, he rejects the partial unity which is the possibility for the experience tokens to be partly unified. In other words, if at an instant $t$, we have two unified tokens $a-b$ on one side and $b-c$ on another, rejection of partial unity is to claim that a and $c$ are also unified. By this rejection, Dainton extends the unity relation to an equivalence relation.

Now, with the help of Dainton's stipulations, an objection arises concerning Bayne's account of unity. Suppose in two successive instants, $E 1$ and $E 2$, we have a common experience token $\left(\mathrm{e}_{2}\right)$, that is, considering $E 1:\left\{\mathrm{e}_{1}, \mathrm{e}_{2}\right\}$ at $t_{1}$ and $E 2:\left\{\mathrm{e}_{2}\right.$, $\left.\mathrm{e}_{3}\right\}$ at $t_{2}=t_{1}+\mathcal{E}$, where $\varepsilon$ is very small. Then, according to the maximal connection conception, $\mathrm{e}_{1}-\mathrm{e}_{2}$ are maximally connected as well as $\mathrm{e}_{2}-\mathrm{e}_{3}$. Consequently, due to the rejection of the partial unity relation, $e_{1}-e_{3}$ should be maximally connected also. Therefore, $\mathrm{e}_{1}-\mathrm{e}_{2}-\mathrm{e}_{3}$ are maximally connected and should be considered as unified, and this means that $E 1$ and $E 2$ should be unified. This is the transitivity of unity from synchronic to diachronic type, which contrasts with Bayne's view (though not with Dainton's).

One possible response to this is that the maximal connection is only applicable to an instant. Since $E 1$ and $E 2$ are not synchronous (and consequently its parts), it is not legitimate to apply it to two experiences occurring at two separate times. Thus, the objection fails. In other words, co-presence is the essential condition of maximal connection, and $\mathrm{e}_{1}-\mathrm{e}_{3}$ are not co-present. To reply, let us first 
make the subjective present and instant concepts clearer. If we admit Retentionalism, then each (successive) subjective instant has some retention of past experiences. In our example, $\mathrm{e}_{1}$ "is" present at $t_{2}$ together with $\mathrm{e}_{3}$ through some retention (as it was supposed that $t_{1}$ and $t_{2}$ are too close). In this way they are co-present and meet the co-presence constraint for maximal connection. On the other hand, if we grant Extensionalism, then again there is a co-presence at least in a specious present range. So, it would be meaningful to apply instantaneous unity to a specious present which includes $e_{1}-e_{2}-e_{3}$. Hence, again, we encounter transitivity in short periods if we admit the specious present ${ }^{4}$.

Hence, from either of the Retentionalist or Extentionalist perspective, the account of Bayne and Dainton for synchronic unity is transitive to the diachronic type, because the relation, as described with the help of maximal connection, permeates from one moment to another.

\subsection{Bayne's Holism and Dainton's C-Holism}

As mentioned earlier, there is a transitivity problem in Bayne's confinement over synchronic unity. Tye's preference for diachronic unity, also, faces the transitivity problem. In sum, how one deals with one type of unity is transitive to the other type. Confinement, either synchronic or diachronic, is problematic. Hence, there is a need for an account covering both types under a single conceptual framework.

Holistic unity is a suitable candidate. Bayne grants some kind of holism for the unity relation which is "to deny that ... elements [of an experience] are independent atoms or units of consciousness" (2010: p. 226). This holism is more clear in the works of Dainton (2006), who considers unity as a co-conscious relation (similar to Shoemaker (2003)) and upon which he introduces the concept of C-holism. Dainton defines the strong type of holism as some part-whole relation in which, if any (sub) part (of an experience) is taken to another whole experience, it results in a different whole experience. He considers two types of properties for experience tokens as parts in a whole: local and global. Local or intrinsic properties ${ }^{5}$ are phenomenal characteristics which identify and individuate experience tokens in different wholes, while global properties are the taste of the whole on the parts. These properties are "phenomenal features gained in virtue of being co-conscious with another experience token" in a whole.

The intrinsic conception of properties in the holistic approach helps with the individuation problem in a unity relation. Tye maintains that since there are no

\footnotetext{
${ }^{4}$ Even when the duration of $e_{1}-e_{2}-e_{3}$ is longer than a specious present period, the problem still exists. Suppose two successive specious presents $E 1$ and $E 2$ have $\mathrm{e}_{2}$ in common. As $\mathrm{e}_{1}-\mathrm{e}_{3}$ are not in the same specious present, it might be claimed that they are not maximally connected. But, according to Dainton's overlap model, the experience token $e_{2}$ in $e_{1}-e_{2}$ and $e_{2}-e_{3}$, in two successive specious present slots $E 1$ and $E 2$, are identical. They are neither numerically different nor at the intensity level. Now, when we consider that $e_{2}$ is maximally connected to $e_{1}$ and $e_{3}$, according to the rejection of partial unity, $e_{1}$ and $e_{3}$ should also be assumed as maximally connected. Therefore, the relation is transitive from one specious present to another.

${ }^{5}$ The other reason Dainton posits some intrinsic or "local" property for the tokens is to evade the commensurability problem.
} 
pure experience tokens, such as pure visual or auditory, there is no room for experience tokens as building blocks of the unified experience. However, with Dainton's whole-part account, it is reasonable to assume that the impure experience tokens (and the instantaneous experiences) are parts of a whole. Then, although by definition, the tokens look impure under the shadow of the global properties inside the whole, they still possess some intrinsic phenomenal characters which identify and individuate them.

On the other hand, the global characteristics of the experience help with extending the conception of unity. According to Dainton, to specify the global character of experience, there is a need to determine which experience tokens are co-conscious with each other. This co-conscious conception can be extended from synchronic unity to diachronic unity. Experience tokens in a synchronic unity, when co-conscious, can be considered as parts of the whole. Similarly, synchronic unities in different moments can be assumed as parts of a diachronic whole. In this way, the part-whole co-conscious approach can embrace both synchronic and diachronic unities.

Nevertheless, the way Dainton describes the co-conscious relation renders the embrace problematic. He maintains that co-conscious experiences cannot be in partial relation but should have a maximal connection (phenomenal proximity) (2006: p. 216). However, for some parts to make a holistic relation, there is no need for all to be maximally connected; that is, there is no need for all pairs of them to be mutually and equally connected. Even if we accept that in the synchronous unity, all parts are connected to each other, in the global unity it is not necessary for all experience tokens to be mutually and equally connected in a period of time. To gain a common phenomenal character, there is no need to have all experiences connected to each other. For example, suppose two sets $E 3=\left\{\mathrm{e}_{1}\right.$, $\left.\mathrm{e}_{2}, \mathrm{e}_{3}\right\}$ and $E 4=\left\{\mathrm{e}_{2}, \mathrm{e}_{4}, \mathrm{e}_{5}\right\}$ at $t_{3}$ and $t_{3}+\varepsilon$, respectively. To have a common phenomenal character attributable to the time domain $t_{3}$ to $t_{3}+\varepsilon$, it is not necessary to have all tokens $e_{1}$ to $e_{5}$ connected to each other. Any selection of members in $E 3$ and $E 4$ can make a global property and thus a unity relation can be gained through any sort of this connection. This entails that synchronic and diachronic unities, in Dainton's proposal, adopt different approaches towards maximal connection, and so are not of the same kind. Thus, they cannot be subsumed by a single rule. I have called this the "dissimilarity problem".

Moreover, the maximal connection in the global (diachronic) sense still suffers from the co-presence problem. How does co-consciousness hold for non-present experiences in the longer periods? How can an experience token have a relation to another token which is not present?

\section{Network Unity}

As aforementioned, attribution of unity over time is problematic. Tye's account focuses on the diachronic type in a way that is transitive to the synchronic type, which he is reluctant to admit. On the other hand, Bayne's confinement to mere 
synchronic unity faces three problems. First, by presuming maximal connection, there would be a transitivity problem from synchronic conception to diachronic type. Second, his tripartite conception is transitive to the extended periods. And third, his account of subjective instant is not accurate. Dainton's co-consciousness, also, fails to offer a similar and consistent account for both synchronic and diachronic unities (the dissimilarity problem). Furthermore, in all accounts, co-presence is a major problem in attribution of unity in the larger periods of time. To resolve mentioned problems around unity attribution over time, I now propose a new model.

The suggestion is to substitute the "maximal connection" with "network" relation; that is, a unified experience can be construed as a network of experience tokens. From now on, I will call this proposed type of unity "network unity". In a network, there are some nodes which can be taken as representatives of experience tokens, and some connections through which each node relates to another. The network conception of unity should be accounted as a holistic approach, since the relationship among a network and its nodes is of a part-whole type. A network cannot be reduced to its nodes. Also, two networks with the same nodes can constitute different wholes by possessing different relations between the nodes. By this model, if we assign a property to every node independent of its relation or connection to other nodes, we can take it as an intrinsic property, which I call "non-relational". Besides, the relations and connections that each node has with others can be interpreted as Dainton's global properties, here called "relational" properties. So, depending on their relations, a node or experience token can have different relational properties in different networks, which is another indicator of the wholeness of a network. Accordingly, in contrast to the maximal connection which necessitates every part (node or token) to be "equally" connected (or directly related) to every other part (mutuality), in the network relation there is no such necessity; instead their connections in the whole make and identify the network.

\subsection{Network Unity and Transitivity Problem}

According to network conception of unity, the representation of synchronic unity changes. In the co-conscious approach, if $e_{1}-e_{2}$ and $e_{2}-e_{3}$ are pair-wise (maximally) connected, then (necessarily) $e_{1}-e_{3}$ are (maximally) connected. But according to the network approach, the only thing needed for these three tokens to constitute a unity relation is to assign a network which encompasses all experience tokens, i.e. $U\left(\mathrm{e}_{1}, \mathrm{e}_{2}, \mathrm{e}_{3}\right)$ in some sorts of relations. The relation between $\mathrm{e}_{1}$ and $e_{2}$ should not necessarily be the same as that between $e_{2}$ and $e_{3}$. Rather, they are both parcels of a single whole network $(U)$. It is more precise to restate the synchronic unity as follows: if $e_{1}$ and $e_{2}$ are members (nodes) of a network (unity relation) and $e_{2}$ and $e_{3}$ are members of the same network, then, $e_{1}, e_{2}$ and $e_{3}$ should all be considered in a single network but not necessarily mutually and equally connected. Therefore, there is no necessity for the supposition of "max- 
imal connection" in the synchronic/diachronic unity, since for any two nodes (token) there should not be a necessary connection in a network. Furthermore, there is no need for these connections to be of the same kind (equal) in a single network. In a similar way, I doubt that all perceived tokens in a physical instant should be involved (mutually) in constructing a unified experience related to that moment. Some empirical evidences, such as the mask experiment or very close successive stimuli, show that all the tokens are not involved in our experience construction.

This is a clear manifestation of what Bayne wants to call subsumption. The holistic network covers all the relations and connections. All parts are involved in a single network in a way that if one of the parts is moved to another network, it constitutes a different whole; the same parts may play different roles in different wholes, even with the same non-relational (intrinsic) properties. Thus, the network approach is aligned with Dainton's strong C-holism. Likewise Dainton's C-holism, this network approach to unity does not have Tye's individuation problem, because, holistically, it is possible to distinguish parts of a whole with the aid of a non-relational and relational property distinction.

Indeed, the network conception is responsible for the "co" prefix in Dainton's "co-conscious" relation. Interestingly, it is associated not only to experience tokens in an instant, but also to the later or former tokens over time. Thus, there is no need to limit ourselves to synchronic unity and then worrying about a leakage to adjacent subjective instants, i.e. the transitivity problem. A holistic network is independent of the temporal grouping of its parts; whether they occur in the same instant or spread over time.

\subsection{Network Unity and Dissimilarity Problem}

However, we should be vigilant about the dissimilarity problem, that is whether the network unity treats both synchronic and diachronic types in the same way. To arm the model in a way that can resist the dissimilarity problem, suppose two subjective instantaneous experiences $E 5=U_{5}\left(\mathrm{e}_{1}, \mathrm{e}_{2}, \mathrm{e}_{3}\right)$ and $E 6=U_{6}\left(\mathrm{e}_{4}, \mathrm{e}_{5}, \mathrm{e}_{6}\right)$ at $t_{5}$ and $t_{6}$, respectively, where these times are not very close. According to the network model, each of $E 5$ and $E 6$ are networks that include their own tokens connected in a one way or another together. Now, suppose a new two-layer network in which some nodes in $E 5$ are connected to some nodes in $E 6$. Then we will have a new two-layer network (in a hierarchical manner) which can be represented as $U_{5,6}(\mathrm{e} 1, \mathrm{e} 2, \mathrm{e} 3, \mathrm{e} 4, \mathrm{e}$, e6); that is, a new unity relation is constructed in which all experience tokens at both times are now networked together. Let us call this network "hierarchical", since the ultimate relation or network is constituted over lower level networks in $E 5$ and $E 6$. This hierarchy lets the network relation be extended for longer periods in the same way that holds for a specific instant ${ }^{6}$. In this way, the network conception can be attributed similarly

${ }^{6}$ Moreover, this hierarchical conception of network unity is aligned with comments of people such as Rosenthal (2003) who represents conscious state as a Higher-Order Thoughts (HOT) and raises objection against common conception of unity which implies maximal connection. 
to both synchronic and diachronic types. Since both are subsumed beneath a single conception of network, the dissimilarity problem is resolved.

This way of representing the unity relation as a network is like the "connectivity view" (Masrour, 2014, forthcoming) insofar that it responds to the fundamental question: "In virtue of what is a unified experience called unified?" The response in the network unity is: when the experiences are parts of a single network. Similarly, the connectivity view, in response, maintains that a set of experiences are unified when there is a "unity path" among them. "Being part of a network" and having a "unity path" can be considered the same when there is a binding relation attached to each two connected nodes of the network; that is, the network constitutes (pair-wised) bound relations. Both connectivity and network views dissent from oneness or, in Masrour's words, the Newtonian conception of unity which seeks for all tokens to come mutually and equally under the single kind of connection. As mentioned earlier, there is no need for all members of an experience set to be connected to each other in the same way. However, the connections in the network conception can be defined differently and so it is more general or universal than the connectivity view. However, this general conception may be criticized as being too liberal; that is, we may legitimately ask for some constraint on the network conception to be counted as a unity relation. In other words, one might say: Is having a network relation sufficient for some set of experience tokens to constitute a unity relation? To respond, the connectivity view might be helpful. The binding relation can introduce one type of constraining conditions over a liberal network unity. However, the specific way of connecting in a network can be referred not only to a single relation between two nodes (like binding relation), but also to the whole network as a general property. For instance, in Tononi's account of concept or experience in "Integrated Information Theory" (IIT) (Oizumi, Albantakis, \& Tononi, 2014), some optimization of information of the system (network) is calculated over a more extended purview of nodes and so the constraining criteria applies to more than a two-node path. In sum, the network conception is a more general conception of unity for which both the connectivity view and IIT can be applied as a constraining condition to limit its broad domain.

\subsection{Network Unity and Co-Presence Problem}

Now, after discussing transitivity and dissimilarity problems, let us examine how the network model works for resolving the co-presence problem; that is, how a relation can be made from relata (experience tokens) which are not present (simultaneously). If we understand the network model in a neuro-physiological framework, and construed it as a neural network, then there would be a line of response. The networks corresponding to different moments can be represented as neural networks which are saved and thus present in the brain as memory. There are some supportive proposals for memory which rely on the networked conjunctive hierarchical model, the best known of which is the Percep- 
tual-Mnemonic Feature-Conjunction Model (PMFC) ((Desimone \& Ungerleider, 1989), (Bussey \& Saksida, 2002) and, more recently, (Cowell, Bussey, \& Saksida, 2006, 2010)). Interestingly, in these models memory and perception are supposed to have the same structure and are, therefore, not understood as distinct faculties in the brain. According to this model, the same structure which is responsible for a subjective instantaneous (diachronic or episodic) experience at the time of perception, also, represents its corresponding memory. Therefore, in an instant, the same hierarchical network which constructs the perceptive experience would later build (a part of) the memory of that perception. According to these proposals, the synchronic unities (networks) at various times (in memory or perception sectors) have the same structure in the same faculty of the brain and thus can come together to construct a single network, i.e. a diachronic unity relation.

\section{Conclusion}

Beyond resolving some conceptual difficulties in unity of consciousness discourse, such as transitivity, dissimilarity, and co-presence problems, the interesting point in uniting the two types of unity under the network conception is that its extension can help to conceive a unified self in all moments of experience. By binding synchronic unity with diachronic one, especially in a unified way, we can achieve a coherent unification that can represent the subjecthood or selfhood. So, the proposal here is that each subject or stream of consciousness might possess a specific way of networked unification which individuates one subject from others. In other words, the specific way of unification which constitutes the network determines or identifies one's "what-it-is-likeness" (Block, 2011). Each subject has a specific/unique way of making networks out of experience tokens. The similarity of unification described for both types of unities permits keeping the same kind of subjecthood in an instant (synchronously) and extends it over time (diachronically); that is, what keeps a subject identified as the same subject before and during different lapses of time is the unique way that one makes networks out of experience tokens and unify them. In this way, the instantaneous subject (self) can be similarly extended or unified with the self in longer periods of time, since their unification process is similar. Future work needs to clarify this association between subjecthood and unification, both conceptually and experimentally (reflected in the brain).

One other potential for the network unity conception is its extension to Higher Order Theories of consciousness. Network can be defined in a hierarchical way; that is, some networks or connections can possess a higher level with respect to others. In the actualist version of Higher Order Thought theory, a phenomenally conscious mental state is the object of a higher-order thought (Carruthers, 2016). Similarly, in the higher-order conception of network unity, some network of experience tokens (or relations between experiences) can be the object (or target) of another network. Accordingly, the higher-order network 
leads to a conscious experience of the lower-order network as the former's object or target. This way of thinking links consciousness with unity. For instance, if we take the lower network as a synchronous unity, then a higher-ordered network of diachronic unity leads to an awareness of synchronous experiences. In this way, although we have not introduced unity as a necessary condition or something bound with being conscious, we have introduced a condition over unity which leads to conscious experience.

By resolving the known problems around attribution of unity of consciousness over time, network unity seems to provide a coherent account for embracing both synchronic and diachronic unities which, as explained, can lead to a clearer understanding of self and subjecthood. This, in turn, sheds light on the connections between unity and consciousness. Future work needs to find constraints on the network conception to provide it with greater predictive power and empirical fruitfulness.

\section{Acknowledgements}

I would like to thank Joseph Levine for his motivations and also constructive comments which greatly improved the manuscript during my research scholarship at University of Massachusetts, Amherst. I also want to show my gratitude to Mehdi Golshani for helpful discussions.

\section{Conflicts of Interest}

The author declares no conflicts of interest regarding the publication of this paper.

\section{References}

Bayne, T., \& Chalmers D. J. (2003). What Is the Unity of Consciousness? In: Cleeremans A., Ed., The Unity of Consciousness: Binding, Integration, and Dissociation. Oxford: Oxford University Press, 23-25.

https://doi.org/10.1093/acprof:oso/9780198508571.003.0002

Bayne, T. (2010). The Unity of Consciousness. New York: Oxford University Press. https://doi.org/10.1093/acprof:oso/9780199215386.001.0001

Block, N. (2011). The Higher Order Approach to Consciousness Is Defunct. Analysis, 71, 419-431. https://doi.org/10.1093/analys/anr037

Broad, C. D. (1923). Scientific Thought. New York: Harcourt, Brace and Co.

Bussey, T.J., \& Saksida, L.M. (2002). The Organization of Visual Object Representations: A Connectionist Model of Effects of Lesions in Perirhinal Cortex. European Journal of Neuroscience, 15, 355-364. https://doi.org/10.1046/j.0953-816x.2001.01850.x

Carruthers, P. (2016). Higher-Order Theories of Consciousness. The Stanford Encyclopedia of Philosophy (Fall 2016 Edition). https://plato.stanford.edu/archives/fall2016/entries/consciousness-higher

Cowell, R. A., Bussey, T. J., \& Saksida, L. M. (2010). Functional Dissociations within the Ventral Object Processing Pathway: Cognitive Modules or a Hierarchical Continuum? Journal of Cognitive Neuroscience, 22, 2460-2479.

https://doi.org/10.1162/jocn.2009.21373 
Cowell, R. A., Bussey, T. J., \& Saksida, L. M. (2006). Why Does Brain Damage Impair Memory? A Connectionist Model of Object Recognition Memory in Perirhinal Cortex. Journal of Neuroscience, 26, 12186-12197. https://doi.org/10.1523/JNEUROSCI.2818-06.2006

Dainton, B. (2006). Stream of Consciousness. London: Routledge.

Desimone, R., \& Ungerleider, L. G. (1989). Neural Mechanisms of Visual Processing in Monkeys. In F. Boller, \& J. Grafman (Eds.), Handbook of Neuropsychology (pp. 267-299). New York: Elsevier Science.

Hempel, C. G., \& Oppenheim, P. (1948). Studies in the Logic of Explanation. In Philosophy of Science (pp. 135-175). https://doi.org/10.1086/286983

Hurley, S. L. (1998). Consciousness in Action. Cambridge, MA: Harvard University Press. William, J. (1890). The Principles of Psychology. New York: Dover.

Kolers, P., \& Grunau, M., (1976) Shape and Color in Apparent Motion. Vision Research, 16, 329-335. https://doi.org/10.1016/0042-6989(76)90192-9

Masrour, F. (2014) Unity, Mereology and Connectivity. Analysis, 74, 509-520. https://doi.org/10.1093/analys/anu064

Masrour, F. (Forthcoming). Unity of Consciousness: In Defense of a Leibnizian View. In C. H. D. Bennett (Ed.), Sensory Integration and the Unity of Consciousness. Cambridge, MA: MIT Press.

Oizumi, M., Albantakis, L., \& Tononi, G. (2014). From the Phenomenology to the Mechanisms of Consciousness: Integrated Information Theory 3.0. PLoS Computational Biology, 10, e1003588. https://doi.org/10.1371/journal.pcbi.1003588

Rosenthal, D. M. (2003). Unity of Consciousness and the Self. Proceedings of the Aristotelian Society, 103, 325-352. https://doi.org/10.1111/j.0066-7372.2003.00075.x

Ruhnau, E. (1995). Time Gestalt and the Observer. In T. Metzinger (Ed.), Conscious EXperience (pp. 165-184). Thorverton: Imprint Academic.

Shoemaker, S. S. (2003). Consciousness and Co-Consciousness. Cleeremans, 1, 59-71.

Tye, M. (2003). Consciousness and Persons. Cambridge, MA: MIT Press. 